\title{
Tumor Cell-Derived Exosomal hsa_circ_0017252 Suppresses the Tumorigenesis of Gastric Cancer Through Suppressing M2 Polarization of Macrophage
}

\section{Jin Song}

Beijing Hospital of Traditional Chinese Medicine

\section{Xiaolong Xu}

Beijing Hospital of Traditional Chinese Medicine

\section{Shasha $\mathrm{He}$}

Beijing Hospital of Traditional Chinese Medicine

Ning Wang

Beijing Hospital of Traditional Chinese Medicine

\section{Yunjing Bai}

Beijing Hospital of Traditional Chinese Medicine

\section{Bo Li ( $\square$ libo@bjzhongyi.com )}

Beijing Hospital of Traditional Chinese Medicine https://orcid.org/0000-0002-2537-2885

\section{Shengsheng Zhang}

Beijing Hospital of Traditional Chinese Medicine

\section{Primary research}

Keywords: GC, exosome, hsa_circ_0017252, miR-17-5p

Posted Date: September 21st, 2021

DOI: https://doi.org/10.21203/rs.3.rs-907938/v1

License: (a) (i) This work is licensed under a Creative Commons Attribution 4.0 International License. Read Full License 


\section{Abstract}

Background: Gastric cancer (GC) is a source of global cancer death. MiR-17-5p is reported to regulate the tumorigenesis of GC. Meanwhile, hsa_circ_0017252 is known to be upregulated in GC. However, the relation between hsa_circ_0017252 and miR-17-5p in GC remains unclear.

Methods: Cell viability, migration and invasion were tested by CCK-8 and transwell assay, respectively. Gene expressions were detected by RT-qPCR, and the protein levels in cells or exosomes were tested by western blot. The efficiency of exosomes isolation was investigated by transmission electron microscope (TEM) and nanoparticle tracking analysis (NTA). Meanwhile, cell apoptosis was tested by flow cytometry. In vivo model was constructed to assess the function of MKN45 cells-derived exosomal hsa_circ_0017252 in GC.

Results: Hsa_circ_0017252 was verified to be significantly downregulated in GC tissues. Hsa_circ_0017252 upregulation significantly decreased the viability and migration of GC cells, and hsa_circ_0017252 could bind with miR-17-5p. Additionally, exosomal hsa_circ_0017252 reversed the polarization of M2 macrophages, and the polarized macrophages decreased the GC cell invasion. Furthermore, exosomes with upregulated hsa_circ_0017252 suppressed the tumor growth of GC.

Conclusion: Delivery of hsa_circ_0017252 by GC cells-derived exosomes inhibits the tumorigenesis of GC through reversing M2 polarization of macrophages. Thus, our finding might provide a new method for GC treatment.

\section{Background}

Gastric cancer (GC) is one of the most frequent cancers globally (1). It stands up for $8 \%$ of cancer incidence all over the world (2). The poor prognosis often results from the distant metastasis, which stands about $90 \%$ of tumor-related deaths (3). Chemotherapy or surgery is the main method for primary tumor treatment, while the therapeutic effect is still not ideal (4). Therefore, it is necessary to find the novel strategies against GC.

MiRNAs are noncoding small RNAs, and they can modulate the mRNA expression by reduction of mRNA translation (5). Moreover, it has been revealed that miRNAs can act as key modulators in various diseases (inflammation and cancers) $(6,7)$. Previous studies showed that miRNAs participate in GC progression. For example, Bao $\mathrm{CH}$ et al revealed that miR-27b-3p could suppress the EMT process in GC through targeting RUNX1 (8). Meanwhile, our previous finding indicated that miR-17-5p could increase the proliferation of GC cells (9). Nevertheless, the association between miR-17-5p and circRNAs in GC remains unclear.

CircRNAs are non-coding RNAs (ncRNAs) which are featured by the covalently closed loop structure (10). Many studies have shown that circRNAs are involved in tumorigenesis of GC via sponging miRNAs. For instance, hsa_circ_0000144 knockdown could suppress the cell growth of GC through mediation of miR- 
502-5p (11); circALPL could promote the progression of GC via sponging miR-127 (12). Meanwhile, it is reported that hsa_circ_0017252 is downregulated in GC (13); however, the association between hsa_circ_0017252 and miR-17-5p in tumorigenesis of GC remains to be further explored.

Exosomes are microvesicles which are derived from multivesicular bodies, and the diameter is 70 to 120 $\mathrm{nm}$ (14). In addition, it has been revealed that exosomes are involved in cell communication through carrying nucleic acids and proteins $(15,16)$. Meanwhile, it has been previously reported that exosomal circRNAs could regulate the tumor progression. For example, exosomal circRNA_102481 could enhance EGFR-TKIs resistance in NSCLC through mediation of miR-30a-5p/ROR1 axis in NSCLC (17); tumorderived exosomal circPSMA1 could facilitate the migration and metastasis in TNBC through regulation of miR-637/Akt1 axis (18). However, the relation between exosomal hsa_circ_0017252 and tumorigenesis of $\mathrm{GC}$ need to be further analyzed.

This study aimed to investigate the relation between hsa_circ_0017252 and miR-17-5p in GC. In addition, the function of exosomal hsa_circ_0017252 in GC was also detected. We hope the research could supply a novel strategy against GC.

\section{Material And Methods}

\section{Cell culture}

GES-1 cells, GC cell lines (MKN-45, AGS and SNU-5), and THP-1 cells were obtained from ATCC and cultured in DMEM containing $10 \% \mathrm{FBS}, 1 \%$ penicillin and streptomycin (Sigma) in the condition of $37^{\circ} \mathrm{C}$ and $5 \% \mathrm{CO}_{2}$.

\section{Clinical sample collection}

In brief, five pairs of GC and adjacent normal tissues were collected from Beijing Hospital of Traditional Chinese Medicine between June 2019 and June 2020. Each tissue sample was stored at $-80^{\circ} \mathrm{C}$. In addition, the serum samples were collected from the patients or healthy people. The present study was approved by the Ethics Committee of Capital Medical University. Meanwhile, the data of the patients with $\mathrm{GC}$ or healthy people were collected with their written informed consent.

\section{Reagents}

IL-13 and IL-4 were bought from MedChemExpress.

\section{Cell transfection}


GC cells were transfected with pcDNA3.1 or pcDNA3.1-hsa_circ_0017252 by using Lipofectamine 2000 for $48 \mathrm{~h}$. After $48 \mathrm{~h}$ of transfection, cells were used in subsequent experiments.

For miRNA transfection, GC cells were transfected with negative control (NC) or miR-17-5p agomir by using Lipofectamine 2000 for $48 \mathrm{~h}$.

\section{Exosome extraction and identification}

Exosomes were extracted from GC cell supernatants by using ultracentrifugation methods. Briefly, the complementary medium was replaced the defined medium (without serum) when cells reached $80 \%$ confluence. The supernatants were harvested and centrifuged after $48 \mathrm{~h}$ of culture. Subsequently, supernatants were collected to extract exosomes by ultracentrifugation (70 min, 120,000 × g, two times). TEM, NTA and western blot were performed to identify the exosomes.

\section{Isolation and cultivation of macrophages}

THP-1 cells were added with PMA $(100 \mathrm{ng} / \mathrm{ml})$. Then, cells were treated with MKN45-Exo, MKN45-Exohsa_circ_0017252 OE or IL4/IL-13 $(20 \mathrm{ng} / \mathrm{ml})$ for $24 \mathrm{~h}$. After that, the CD206 (macrophages surface maker) and CD86 (macrophages surface maker) were tested by flow cytometry.

\section{Fluorescence in situ hybridization}

The co-localization of hsa_circ_0017252 and miR-17-5p in the cytoplasm was observed by using FISH (19).

\section{RT-qPCR}

TRIzol® reagent was applied to extract total RNA. PrimeScript RT reagent kit was used in reverse transcription. Then, SYBR premix Ex Taq II kit (Takara) was used in RT-qPCR, and Real-Time qPCRs were used three times: 2 minutes at $94^{\circ} \mathrm{C}$, followed by 35 cycles $\left(94^{\circ} \mathrm{C}\right.$ for $30 \mathrm{~s}$ and $55^{\circ} \mathrm{C}$ for $\left.45 \mathrm{~s}\right)$. The primer sequences were listed as follows: hsa_circ_0017252 forward, 5'-AAACUACUAGGUAAAGGCACUUUU-3' and reverse, 5'-GATAAAUUUAAAGCUCUGUGCC-3'; $\beta$-actin forward, 5'-GTCCACCGCAAATGCTTCTA-3' and reverse, $5^{\prime}$-TGCTGTCACCTTCACCGTTC-3'. The data were quantified by using $2^{-\Delta \triangle t}$ method. $\beta$-actin was regarded as internal control.

\section{CCK-8 assay}

GC cells ( $5 \times 10^{3}$ cells/well) were treated for $48 \mathrm{~h}$ as follows: NC, hsa_circ_0017252 OE or hsa_circ_0017252 OE + miR-17-5p agomir. Subsequently, GC cells were added with CCK-8 (10 $\mu$ l) for 
another $2 \mathrm{~h}$. The absorbance of each well $(450 \mathrm{~nm})$ was assessed using a microplate reader.

\section{Immunofluorescence}

GC cells were fixed and then incubated with anti-PKH26 $(1: 1,000)$ or anti-CD206 $(1: 1,000)$. Subsequently, the secondary antibody $(1: 5,000$; goat anti-rabbit $\lg G)$ was used to incubate GC cells. Finally, the data was observed by a microscope.

\section{Western blotting}

RIPA was applied to extract protein from tissues or cell lines. BCA kit was applied to quantify the total protein. SDS-PAGE (10\%) was applied to separate the proteins (40 $\mu \mathrm{g}$ per lane), and then proteins were transferred onto PVDF membranes. Subsequently, the membranes were incubated overnight at $4^{\circ} \mathrm{C}$ with primary antibodies targeted against: CD63 (1:1,000), TSG101 (1:1,000), Runx3 (1:1,000), a-SMA (1:1,000), vimentin $(1: 1,000), \mathrm{N}$-cadherin $(1: 1,000)$, E-cadherin $(1: 1,000)$ and $\beta$-actin $(1: 1,000)$ after blocked with skimmed milk (5\%) for $1 \mathrm{~h}$. Following primary incubation, HRP-conjugated secondary antibodies $(1: 5,000)$ were used to incubate the membranes for $1 \mathrm{~h}$. ECL kit was used to visualize the protein bands. $\beta$-actin was regarded as internal control. The densitometry analysis was performed by using IPP 6.0 (Image-Pro Plus 6.0).

\section{Transwell assay}

Matrigel ( $100 \mu \mathrm{l}$, not included in migration assay) was used to pre-treat the upper chamber. GC cells $\left(1.0 \times 10^{6}\right.$ cells per chamber) were seeded into the upper chamber in medium ( $1 \%$ FBS). In addition, the lower chamber was supplemented with RPMI1640 (10\% FBS). Subsequently, the chamber was rinsed and fixed at $4^{\circ} \mathrm{C}$. Then, crystal violet $(0.1 \%)$ was used to stain the chamber for 20 minutes. The data was observed under a microscope after the chamber was washed.

\section{Dual luciferase reporter assay}

Hsa_circ_0017252 containing the binding sites of miR-17-5p was cloned into the pGL6 vectors for establishment of hsa_circ_0017252 (WT/MT). Hsa_circ_0017252 (WT/MT) was transfected into GC cells with miR-17-5p/NC mimics using Lipofectamine 2000. Dual-Glo Luciferase Assay System was used to analyze the result.

\section{Cell apoptosis detection}


GC cells $\left(5 \times 10^{4}\right)$ were centrifuged and resuspended. Subsequently, cells were treated with Annexin V-FITC and $\mathrm{Pl}$ at $4^{\circ} \mathrm{C}$ for $15 \mathrm{~min}$. Cell apoptosis was analyzed by flow cytometer, and FlowJo was used to quantify the data.

\section{ELISA}

IL-10 and IL-1 $\beta$ levels in GC cell supernatants were assessed by ELISA kit.

\section{In vivo study}

BALB/c nude mice (aged 6-8 weeks old, $n=24$ ) were placed in a room with dedicated SPF facility. MKN45 cells co-cultured with macrophages-exo, macrophages or macrophages ${ }^{\text {hsa_circ_0017252 OE }}$ were transplanted in each mouse subcutaneously. The tumor volume was measured weekly as the flowing equation: length $x$ width $^{2}$. Finally, mice were sacrificed for tissue collection. Then, tumors were weighted. All the experiments were performed in line with NIH guide. Moreover, the Ethics Committees of Capital Medical University approved the protocol of this study.

\section{Statistical analysis}

Three independent experiments were performed in each group. In addition, the mean \pm standard deviation (SD) was used to express all data. The comparisons between two groups were analyzed using Student's $\mathrm{t}$-test, and the differences between multiple groups (more than 2 groups) were analyzed by one-way analysis of variance (ANOVA) followed by Tukey's test (Graphpad Prism7). P<0.05 indicates a significant change.

\section{Results}

\section{Hsa_circ_0017252 bound with miR-17-5p in gastric cancer cells}

To investigate the relation between hsa_circ_0017252 and miR-17-5p, starbase and dual luciferase assay was performed. As revealed in Figure 1A, miR-17-5p was predicted to be the downstream miRNA of hsa_circ_0017252. The relative luciferase activity in WT-hsa_circ_0017252 was significantly downregulated by miR-17-5p mimics, while miR-17-5p mimics limitedly affected the luciferase activity in Mut-hsa_circ_0017252 (Figure 1B). The background information of hsa_circ_0017252 was listed in Supplementary Figure 1A, and hsa_circ_0017252 level in gastric cancer cells was obviously downregulated, compared with that in GES-1 cells (Figure 1C). Consistently, hsa_circ_0017252 level in GC tissues or the serum of patients with GC was significantly downregulated, compared with control (Figure 
1D and 1E). Meanwhile, hsa_circ_0017252 was found to be co-localized with miR-17-5p in cytoplasm (Figure 1F). In summary, hsa_circ_0017252 bound with miR-17-5p in GC cells.

\section{Hsa_circ_0017252 overexpression significantly induced GC cell apoptosis via sponging miR-17-5p}

In order to further confirm the relation between hsa_circ_0017252 and miR-17-5p in gastric cancer cells, gastric cancer cells were treated with pcDNA3.1-hsa_circ_0017252. Then, RT-qPCR was performed. As revealed in Figure 2A, hsa_circ_0017252 expression in AGS and MKN45 cells was significantly upregulated by pcDNA3.1-hsa_circ_0017252. Additionally, hsa_circ_0017252 overexpression obviously suppressed the viability of gastric cancer cells, while miR-17-5p mimics reversed this phenomenon (Figure 2B and 2C). Moreover, miR-17-5p mimics significantly reversed hsa_circ_0017252 overexpressioninduced cell apoptosis (Figure 2D and 2E). Taken together, hsa_circ_0017252 significantly induced the apoptosis in gastric cancer cells via sponging miR-17-5p.

\section{Hsa_circ_0017252 inhibited the migration of GC cells}

To detect the impact of hsa_circ_0017252 on gastric cancer cell migration, transwell assays were used. The data demonstrated hsa_circ_0017252 overexpression greatly inhibited GC cell migration, which was restored by miR-17-5p mimics (Figure 3A and 3B). Meanwhile, hsa_circ_0017252 upregulation greatly increased the expressions of RUNX3, E-cadherin and inhibited the protein levels of N-cadherin and a-SMA (Figure 3C and 3D). However, the effect of hsa_circ_0017252 overexpression on these proteins was greatly inhibited by miR-17-5p agomir (Figure 3C and 3D). Thus, it can be concluded that hsa_circ_0017252 upregulation inhibited GC cell migration through binding with miR-17-5p. Since MKN45 cells were more sensitive to hsa_circ_0017252 treatment, MKN45 cells were selected of use in subsequent analysis.

\section{Exosomes transferred hsa_circ_0017252 from GC cells to macrophages}

Exosomes from tumors were reported to play crucial roles in tumor progression $(7,20)$. Thereby, exosomes were isolated from MKN45 cells, and then the efficiency of isolation was verified by TEM. As shown in Figure 4A, the result of TEM indicated that the diameter of rounded particles was from 30 to $150 \mathrm{~nm}$, and the similar size distribution of exosomes was also observed after NTA detection (Supplementary Figure 1B and 1C). Moreover, exosomal proteins (CD63 and TSG101) were highly expressed in exosomes from MKN45 cells that were treated with pcDNA3.1-hsa_circ_0017252

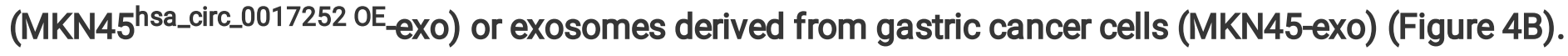




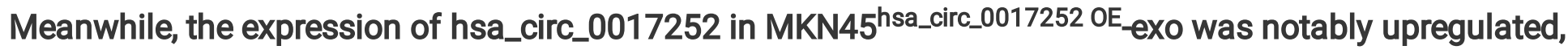
than that in MKN45-exo (Figure 4C).

To detect whether exosomes can transmit hsa_circ_0017252 between MKN45 cells and macrophages, macrophages were co-cultured with PKH26-labeled exosomes derived from GC cells. In addition, PKH26 lipid dye were indicated in macrophages after co-culture for $48 \mathrm{~h}$ (Figure 4D). This result suggested that

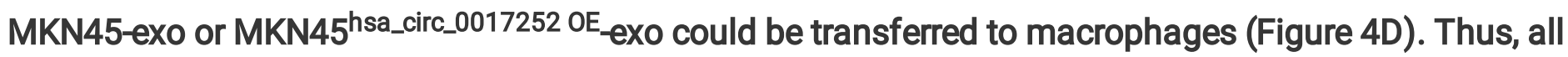
these data suggested that exosomes could transfer hsa_circ_0017252 from GC cells to macrophages.

\section{Exosomal hsa_circ_0017252 suppressed M2 polarization in macrophages}

For the purpose of investigating the role of MKN45 ${ }^{\text {hsa_circ_ } 0017252}$ OE exo in M2 polarizatio, macrophages were added with MKN45 cell-derived exosomes $(50 \mu \mathrm{g} / \mathrm{ml})$ or IL-4/IL-13. The data revealed a CD86 low $_{\text {/CD206 }}$ high phenotype in cells after treated with MKN45-exo, while this phenomenon was

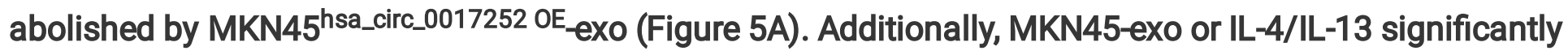
decreased the level of IL-1 $\beta$ and upregulated the level of IL-10 in macrophages; however, the effect of MKN45-exo on IL-10 and IL-1 $\beta$ was significantly rescued by MKN45 ${ }^{\text {hsa_circ_0017252 OE }}$ exo (Figure 5B and 5C). To sum up, exosomal-hsa_circ_0017252 could decrease M2 macrophage polarization.

\section{Exosomal hsa_circ_0017252 significantly suppressed GC cell migration and invasion through inhibiting M2 macrophage polarization}

To confirm whether exosomal hsa_circ_0017252 could suppress the invasion and migration of gastric cancer cells by suppressing M2 macrophages polarization, THP-1 cells were added with PMA (100 $\mathrm{ng} / \mathrm{ml}$ ) and co-cultured with exosomes derived from MKN45 cells for $24 \mathrm{~h}$ (Figure 6A). As shown in Figure 6B, the level of hsa_circ_0017252 in conditioned medium (CM) of macrophages/hsa_circ_0017252 was not affected by RNase A, while it was greatly suppressed in the presence of RNase A plus Triton X-100. Moreover, the invasion and migration of MKN45 cells were significantly increased by MKN45-exo-induced M2 macrophages; however, the effect of M2 macrophages was significantly restored by exosomal hsa_circ_0017252 (Figure 6C). Exosomal hsa_circ_0017252 reversed the effect of macrophages treated with MKN45-exo on a-SMA, E-cadherin and N-cadherin levels (Figure 6D-6G). Taken together, exosomal hsa_circ_0017252 significantly inhibited the invasion and migration of gastric cancer cells through decreasing M2 macrophage polarization. 


\section{Exosomal hsa_circ_0017252 suppressed the tumor growth of GC through inhibiting the M2 polarization in macrophages}

To further detect the function of exosomal hsa_circ_0017252 in GC in vivo, in vivo model was constructed. As revealed in Figure 7A-7C, MKN45-exo-stimulated macrophages notably enhanced the tumor weight and tumor volume of mice, while macrophages treated with MKN45 ${ }^{\text {hsa_circ_0017252 OE }}$ exo restored these phenomena. Positive CD206 rate in tissues was greatly inhibited in MKN45 + $M^{\text {hsa_circ_0017252 OE }}$ group, than MKN45 + $\mathrm{M}^{\mathrm{Exo}}$ group. (Figure 7D). Moreover, MKN45-exo-incubated macrophages greatly elevated the level of vimentin and inhibited the expressions of E-cadherin and

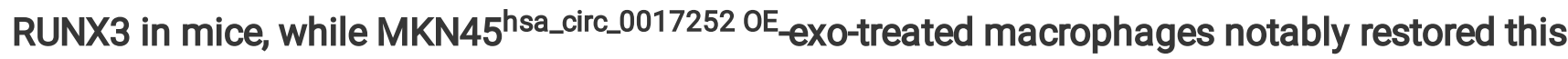
phenomenon (Figure 7E). In summary, exosomal hsa_circ_0017252 could attenuate the growth of GC in vivo through inhibiting the $\mathrm{M} 2$ polarization in macrophages.

\section{Discussion}

Exosomes are derived from multiple cells (including the cancer cells), and it has been reported that exosomes are associated with cancer development (21-23). Some studies showed that exosomes play a critical role in tumor microenvironment, and they can promote the tumorigenesis of cancer through establishing the relation between tumor cells and stromal cells $(7,20)$. In addition, it has been indicated that M2 macrophages are closely correlated with the tumor progression as they always promote the recurrence and metastasis of malignant tumor $(24,25)$. In our research, exosomes derived from GC cells could induce the macrophage M2 polarization, and the polarized macrophages promoted the invasion and migration of GC cells. Moreover, we found exosomes derived from GC cells are important mediators in tumor microenvironment and they can play a vital role in association among different cells.

It has been revealed that miR-17-5p could act as a promoter in $\mathrm{GC}(9,26)$. In addition, our research indicated that hsa_circ_0017252 could upregulate the expression of RUNX3 via binding with miR-17-5p, and this finding was similar to our previous work (9). Meanwhile, Zhao J et al found that PDCD4 was the direct target of miR-17-5p in GC.(26) PDCD4 was known to be a suppressor in tumor progression $(27,28)$, and RUNX3 could inhibit the cell growth in cancer $(29,30)$. Thus, the similar function between PDCD4 and RUNX3 might result in Zhao $\mathrm{J}$ et al and our study.

CircRNA dysregulation often cause cancer occurrence $(19,31)$. This study firstly explored the function of hsa_circ_0017252 in GC. In addition, hsa_circ_0017252 level was decreased in exosomes, and it could decrease GC cell migration. hsa_circ_0017252 upregulation in exosomes suppressed M2 macrophage polarization, which further attenuated the invasion, migration and EMT process of GC cells. Based on the above data, it can be concluded that GC cell-derived exosomal hsa_circ_0017252 could act as a crucial mefiator in tumor metastasis and tumor microenvironment. 
Obviously, there are some weaknesses in this work as follows: 1) more signaling pathways related with the tumorigenesis of GC need to be further explored; 2 ) more downstream targets of miR-17-5p are needed to be investigated; 3 ) the function of exosomal miR-17-5p in GC remains unclear. Hence, more analysis are needed in future.

Tumor cell-derived exosomal hsa_circ_0017252 inhibits the tumorigenesis of GC through suppressing M2 polarization of macrophage. Thus, our study shed new lights on exploring the therapeutic methods against GC.

\section{Abbreviations}

GC gastric cancer

Exo exosome

Circular RNAs circRNAs

MicroRNAs miRNAs

\section{Declarations}

Ethics approval and consent to participate

The present study was approved by the Ethics Committee of Capital Medical University (No.

CMU20210402). Meanwhile, the data of the patients with GC or healthy people were collected with their written informed consent. In addition, the Ethics Committees of Capital Medical University approved the protocol of in vivo study (No. CMU20210405).

Consent for publication

Not applicable.

Availability of data and materials

The datasets analyzed during the current study are available from the corresponding author on reasonable request.

Author contribution

Bo Li and Shengsheng Zhang conceived and supervised the study. Jin Song, Xiaolong Xu and Shasha He designed the study. Ning Wang and Yunjing Bai performed the experiments and analyzed the data. All authors reviewed the results and approved the final version of the manuscript. 
Not applicable

Funding

None

Conflict of interests

These authors declared no competing interests in this research.

\section{References}

1. Zarin B, Eshraghi A, Zarifi F, Javanmard SH, Laher I, Amin B, et al. A review on the role of Tau and Stathmin in gastric cancer metastasis. Eur J Pharmacol. 2021:174312.

2. Du S, Fang Z, Ye L, Sun H, Deng G, Wu W, et al. Pretreatment neutrophil-to-lymphocyte ratio predicts the benefit of gastric cancer patients with systemic therapy. Aging (Albany NY). 2021;13.

3. Osterkamp J, Strandby RB, Nerup N, Svendsen MBS, Svendsen LB, Achiam MP. Time to maximum indocyanine green fluorescence of gastric sentinel lymph nodes and feasibility of combined indocyanine green/sodium fluorescein gastric lymphography. Langenbecks Arch Surg. 2021.

4. Khaled I, Priego P, Soliman H, Faisal M, Saad Ahmed I. Oncological outcomes of laparoscopic versus open gastrectomy after neoadjuvant chemotherapy for locally advanced gastric cancer: a retrospective multicenter study. World J Surg Oncol. 2021;19:206.

5. Srivastava S, Suprasanna P. MicroRNAs: Tiny, powerful players of metal stress responses in plants. Plant Physiol Biochem. 2021;166:928-38.

6. Wu Q, Chen Q, Wang J, Fan D, Zhou H, Yuan Y, et al. Long non-coding RNA Pvt1 modulates the pathological cardiac hypertrophy via miR-196b-mediated OSMR regulation. Cell Signal. 2021:110077.

7. Moradi-Chaleshtori M, Shojaei S, Mohammadi-Yeganeh S, Hashemi SM. Transfer of miRNA in tumorderived exosomes suppresses breast tumor cell invasion and migration by inducing M1 polarization in macrophages. Life Sci. 2021:119800.

8. Bao CH, Guo L. miR-27b-3p Inhibits Invasion, Migration and Epithelial-mesenchymal Transition in Gastric Cancer by Targeting RUNX1 and Activation of the Hippo Signaling Pathway. Anticancer Agents Med Chem. 2021.

9. Song J, Liu Y, Wang T, Li B, Zhang S. MiR-17-5p promotes cellular proliferation and invasiveness by targeting RUNX3 in gastric cancer. Biomed Pharmacother. 2020;128:110246.

10. Zhao Y, Guo H, Wang W, Zheng G, Wang Z, Wang X, et al. High-throughput screening of circRNAs reveals novel mechanisms of tuberous sclerosis complex-related renal angiomyolipoma. Hum Genomics. 2021;15:43.

11. Gao H, Xu J, Qiao F, Xue L. Depletion of hsa_circ_0000144 Suppresses Oxaliplatin Resistance of Gastric Cancer Cells by Regulating miR-502-5p/ADAM9 Axis. Onco Targets Ther. 2021;14:2773-87. 
12. Wu P, Ye D, Li J, Yan F, Jin X, Zhang Z, et al. circALPL Sponges miR-127 to Promote Gastric Cancer Progression by Enhancing MTDH Expression. J Cancer. 2021;12:4924-32.

13. Wu L, Wang J, He X, Zhu Y, Jiang $X$, Chen $Y$, et al. Deep learning system compared with expert endoscopists in predicting early gastric cancer and its invasion depth and differentiation status (with videos). Gastrointest Endosc. 2021.

14. Zhang J, Li S, Li L, Li M, Guo C, Yao J, et al. Exosome and exosomal microRNA: trafficking, sorting, and function. Genomics Proteomics Bioinformatics. 2015;13:17-24.

15. Joyce DP, Kerin MJ, Dwyer RM. Exosomeencapsulated microRNAs as circulating biomarkers for breast cancer. Int J Cancer. 2016;139:1443-8.

16. Zhang Y, Bi J, Huang J, Tang Y, Du S, Li P. Exosome. A Review of Its Classification, Isolation Techniques, Storage, Diagnostic and Targeted Therapy Applications. Int J Nanomedicine. 2020;15:6917-34.

17. Yang B, Teng F, Chang L, Wang J, Liu DL, Cui YS, et al. Tumor-derived exosomal circRNA_102481 contributes to EGFR-TKIs resistance via the miR-30a-5p/ROR1 axis in non-small cell lung cancer. Aging. 2021;13:13264-86.

18. Yang SJ, Wang DD, Zhong SL, Chen WQ, Wang FL, Zhang J, et al. Tumor-derived exosomal circPSMA1 facilitates the tumorigenesis, metastasis, and migration in triple-negative breast cancer (TNBC) through miR-637/Akt1/beta-catenin (cyclin D1) axis. Cell Death Dis. 2021;12:420.

19. Liu B, Tian Y, Chen M, Shen H, Xia J, Nan J, et al. CircUBAP2 Promotes MMP9-Mediated Oncogenic Effect via Sponging miR-194-3p in Hepatocellular Carcinoma. Front Cell Dev Biol. 2021;9:675043.

20. Zhou D, Xia Z, Xie M, Gao Y, Yu Q, He B. Exosomal long non-coding RNA SOX2 overlapping transcript enhances the resistance to EGFR-TKIs in non-small cell lung cancer cell line H1975. Hum Cell. 2021.

21. Wang Y, Lin C. Exosomes. miR-22-3p Derived from Mesenchymal Stem Cells Suppress Colorectal Cancer Cell Proliferation and Invasion by Regulating RAP2B and PI3K/AKT Pathway. J Oncol. 2021;2021:3874478.

22. Li T, Tao Z, Zhu Y, Liu X, Wang L, Du Y, et al. Exosomal annexin A6 induces gemcitabine resistance by inhibiting ubiquitination and degradation of EGFR in triple-negative breast cancer. Cell Death Dis. 2021;12:684.

23. Majumdar R, Tavakoli Tameh A, Arya SB, Parent CA. Exosomes mediate LTB4 release during neutrophil chemotaxis. PLoS Biol. 2021;19:e3001271.

24. Xun J, Du L, Gao R, Shen L, Wang D, Kang L, et al. Cancer-derived exosomal miR-138-5p modulates polarization of tumor-associated macrophages through inhibition of KDM6B. Theranostics. 2021;11:6847-59.

25. Yuan $Y$, Wang Z, Chen M, Jing Y, Shu W, Xie Z, et al. Macrophage-Derived Exosomal miR-31-5p Promotes Oral Squamous Cell Carcinoma Tumourigenesis Through the Large Tumor Suppressor 2Mediated Hippo Signalling Pathway. J Biomed Nanotechnol. 2021;17:822-37.

26. Zhao J, Xiao A, Liu C, Ye C, Yin K, Lu M, et al. The HIF-1A/miR-17-5p/PDCD4 axis contributes to the tumor growth and metastasis of gastric cancer. Signal Transduct Target Ther. 2020;5:46. 
27. Filippova EA, Fridman MV, Burdennyy AM, Loginov VI, Pronina IV, Lukina SS, et al. Long Noncoding RNA GAS5 in Breast Cancer: Epigenetic Mechanisms and Biological Functions. Int J Mol Sci. 2021;22.

28. Egusquiza-Alvarez CA, Castaneda-Patlan MC, Albarran-Gutierrez $\mathrm{S}$, Gonzalez-Aguilar $\mathrm{H}$, MorenoLondono AP, Maldonado V, et al. Overexpression of Multifunctional Protein p32 Promotes a Malignant Phenotype in Colorectal Cancer Cells. Front Oncol. 2021;11:642940.

29. Liu W, Tan S, Bai X, Ma S, Chen X. Long non-coding RNA LINC01215 promotes epithelialmesenchymal transition and lymph node metastasis in epithelial ovarian cancer through RUNX3 promoter methylation. TransI Oncol. 2021;14:101135.

30. Su H, Fan G, Huang J, Qiu X. YBX1 regulated by Runx3-miR-148a-3p axis facilitates non-small-cell lung cancer progression. Cell Signal. 2021;85:110049.

31. Ameli-Mojarad M, Ameli-Mojarad M, Nourbakhsh M, Nazemalhosseini-Mojarad E. Circular RNA hsa_circ_0005046 and hsa_circ_0001791 May Become Diagnostic Biomarkers for Breast Cancer Early Detection. J Oncol. 2021;2021:2303946.

\section{Figures}

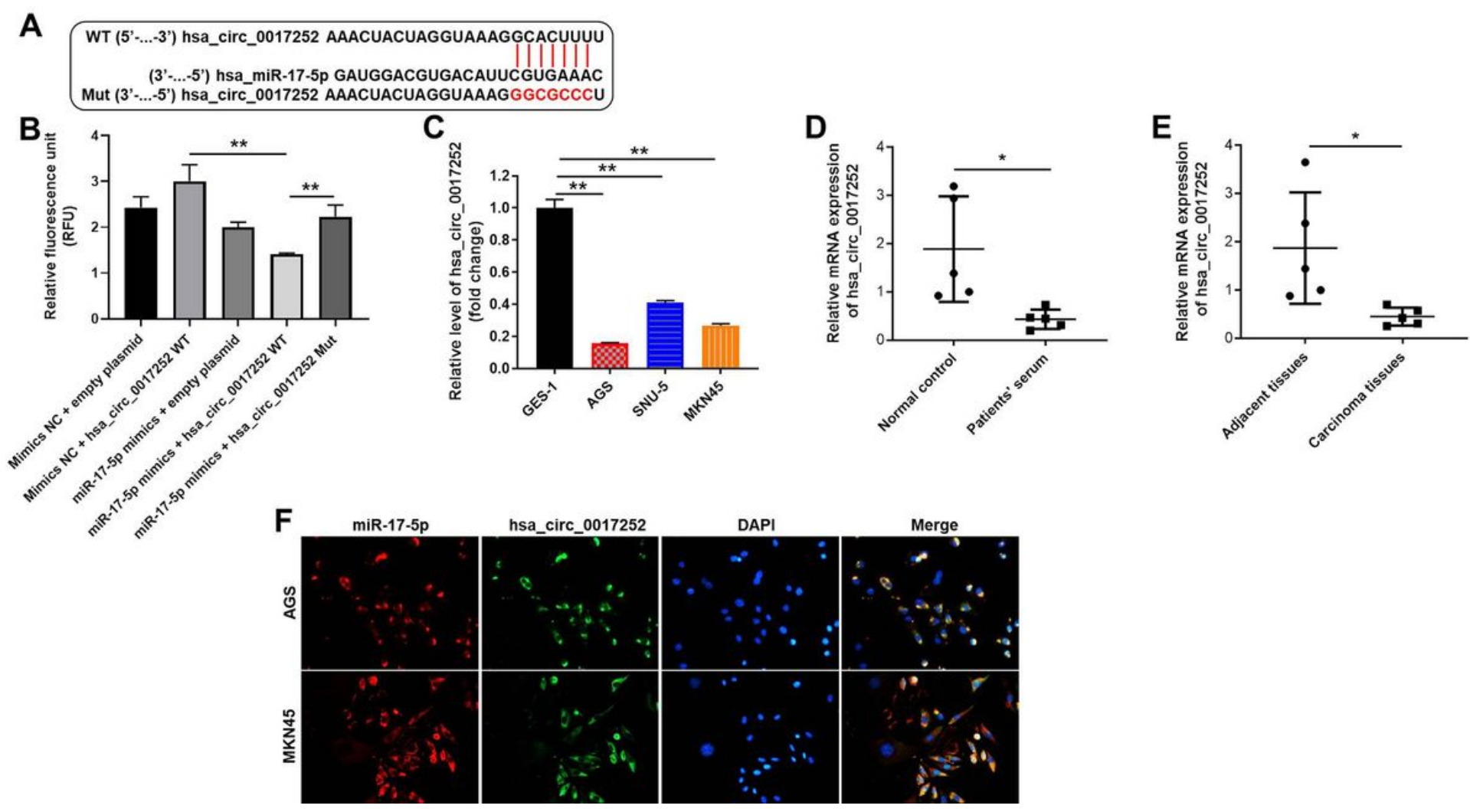

Figure 1

Hsa_circ_0017252 bound with miR-17-5p in GC cells. (A) The binding sites between hsa_circ_0017252 and miR-17-5p were predicted by starbase. (B) The relative luciferase activity in WT/MUT- 
hsa_circ_0017252 was detected by dual luciferase report assay. (C) Hsa_circ_0017252 expression in GES1, AGS, SNU-5 and MKN45 cells was investigated by RT-qPCR. (D) Hsa_circ_0017252 level in serum of normal people or patients with GC was investigated by RT-qPCR. (E) Hsa_circ_0017252 expression in GC or adjacent normal tissues was tested by RT-qPCR. (F) The co-localization between hsa_circ_0017252 and miR-17-5p was investigated by FISH. *P<0.05, **P<0.01.
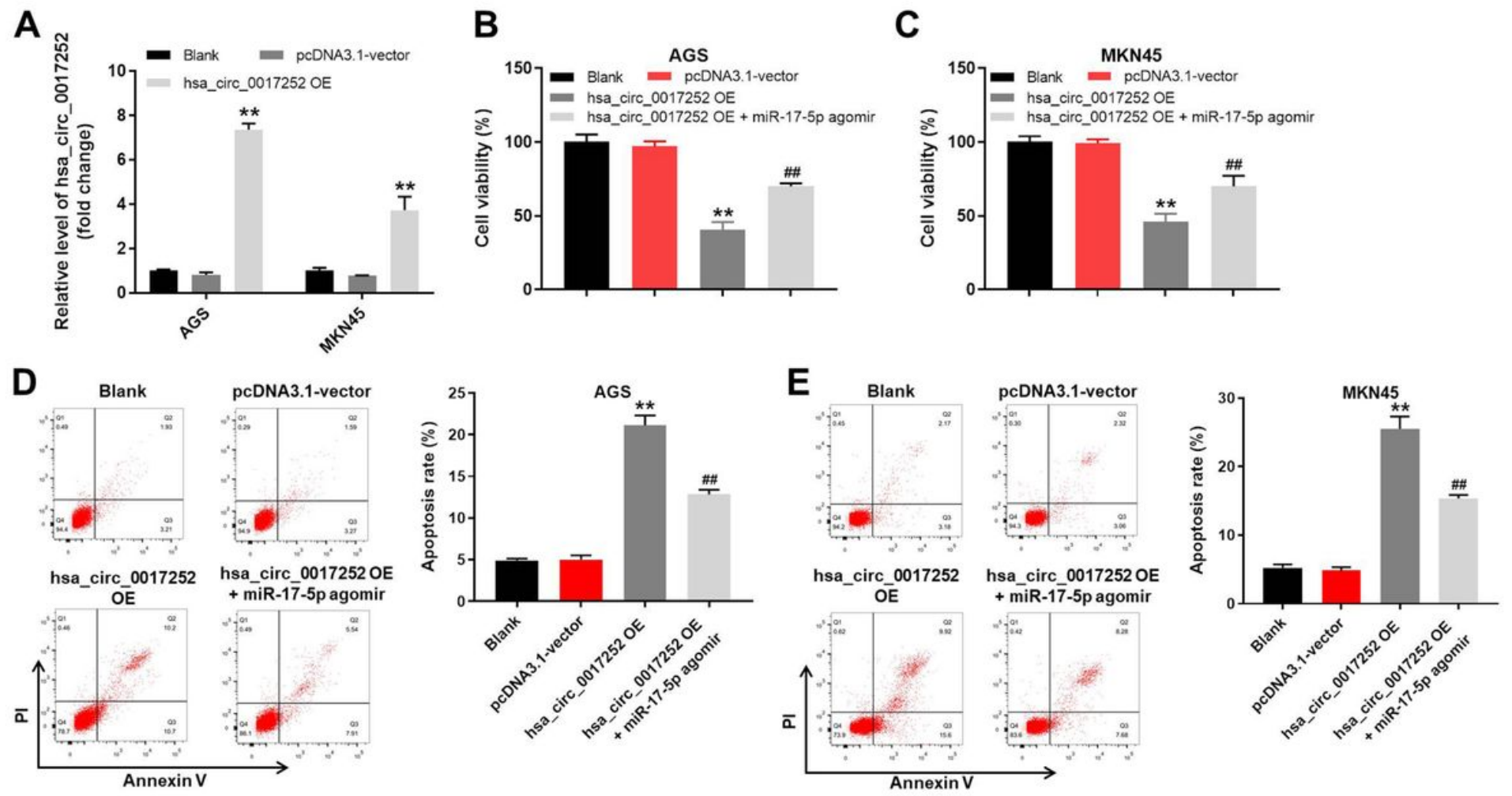

Figure 2

Hsa_circ_0017252 upregulation significantly induced the apoptosis in gastric cancer cells via sponging miR-17-5p. (A) GC cells were transfected with pcDNA3.1 or pcDNA3.1-hsa_circ_0017252. Hsa_circ_0017252 expression in GC cells was detected by RT-qPCR. (B, C) AGS or MKN45 cells were treated with pcDNA3.1, pcDNA3.1-hsa_circ_0017252 or pcDNA3.1-hsa_circ_0017252 + miR-17-5p agomir. The cell viability was tested by CCK-8 assay. (D, E) The apoptosis of GC cells was investigated by flow cytometry. ${ }^{* *} \mathrm{P}<0.01$ compared with blank. \#\#P<0.01 compared with hsa_circ_0017252 OE. 

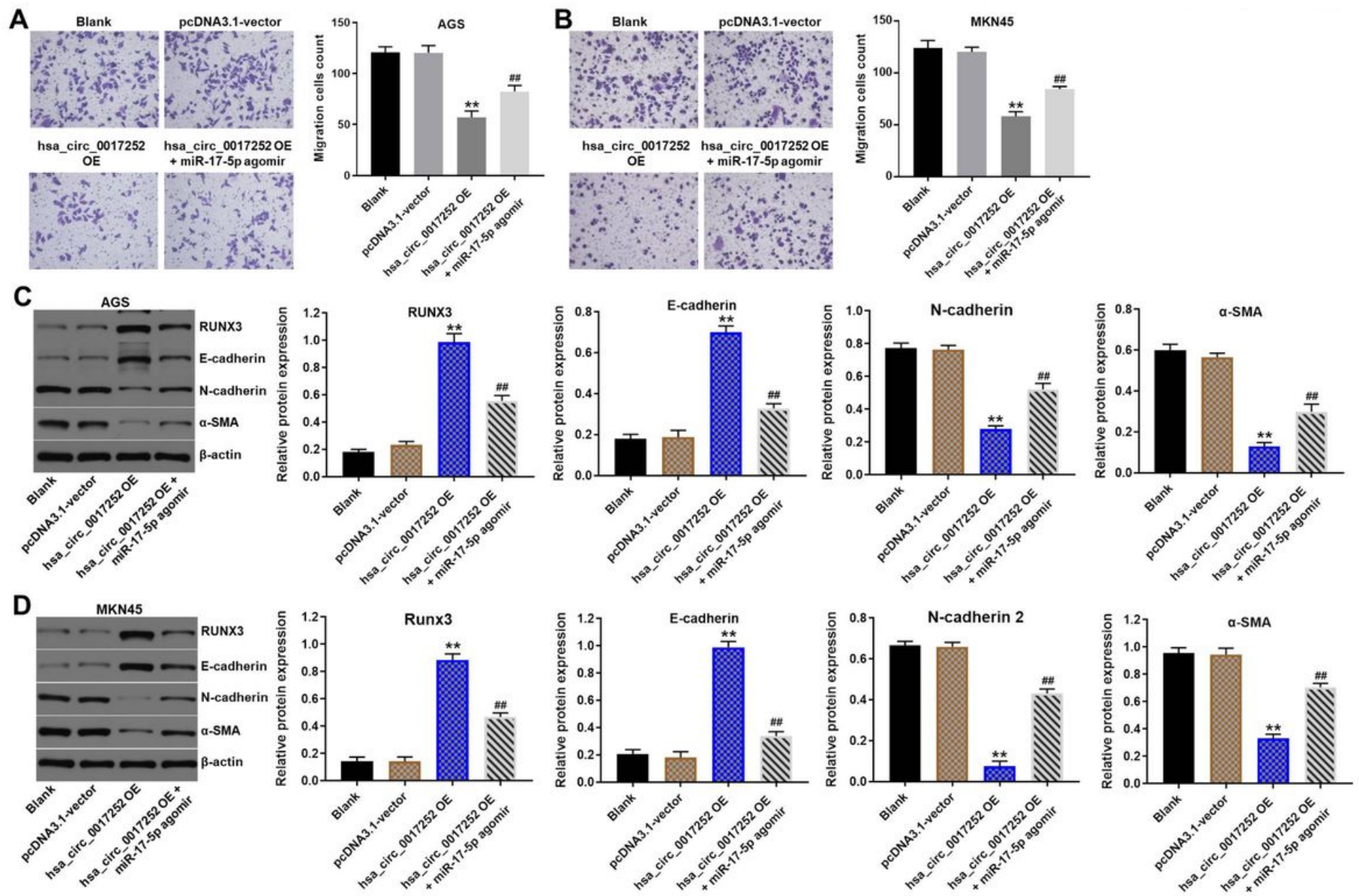

Figure 3

Overexpression of hsa_circ_0017252 inhibited the migration of gastric cancer cells. (A, B) The migration of GC cells was detected by transwell assay. (C, D) E-cadherin, RUNX3, N-cadherin and a-SMA levels in GC cells were investigated by western blot. $\beta$-actin was used for normalization. $* * P<0.01$ compared with blank. \#\#P<0.01 compared with hsa_circ_0017252 OE. 


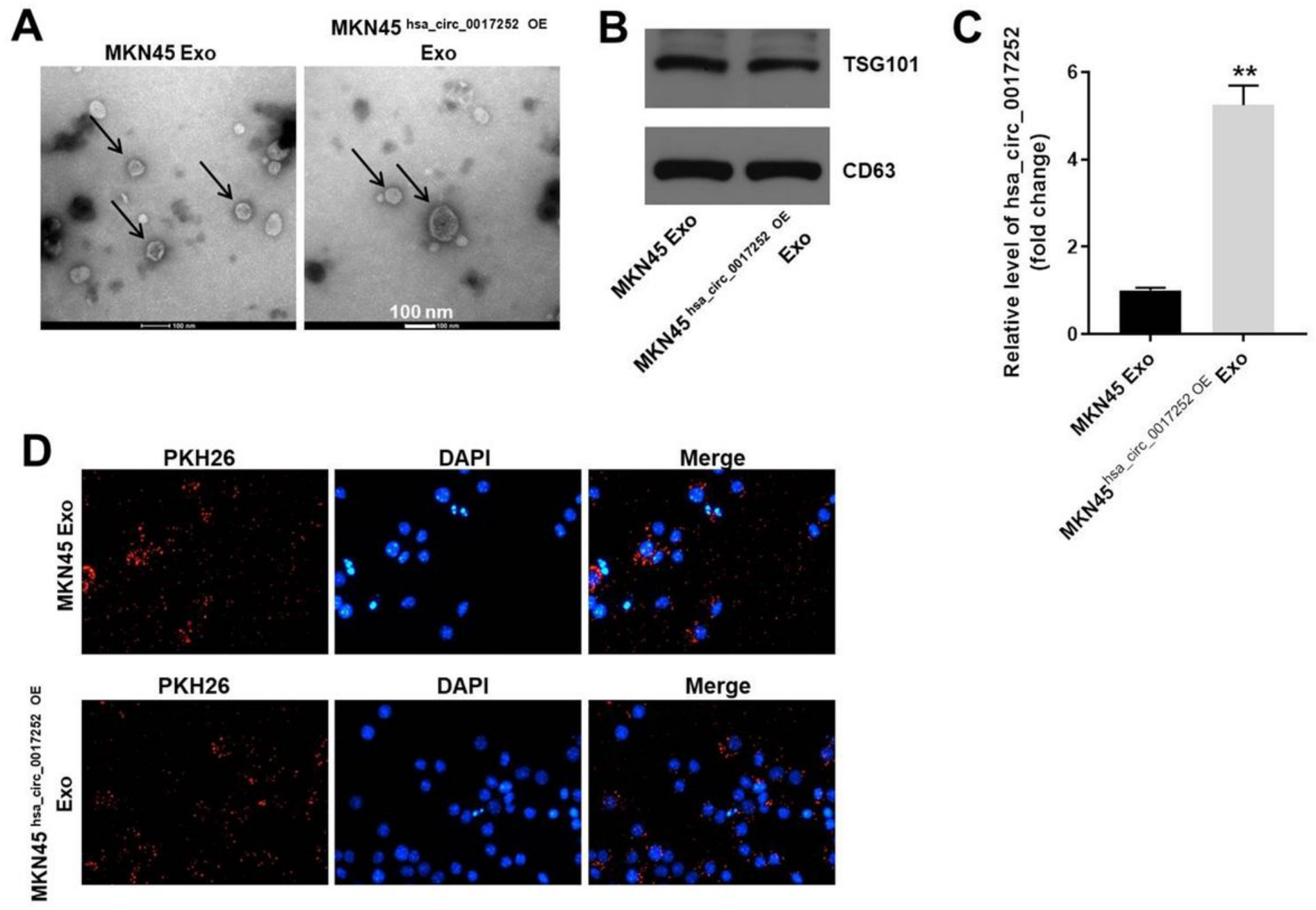

Figure 4

Hsa_circ_0017252 can be transferred from MKN45 cells to macrophages cells via exosomes. (A) The efficiency of exosome isolation was tested by TEM. Black arrows indicate the exosomes. (B) The expressions of TSG101 and CD63 in MKN45-exo or MKN45 hsa_circ_0017252-OE-exo were investigated by western blot. (C) Hsa_circ_0017252 level in MKN45-exo or MKN45 hsa_circ_0017252-0E-exo was assessed by RT-qPCR. (D) THP-1 cells were added with PMA (100 ng/ml) and then added with exosomes derived from GC cells. Fluorescence staining was used to investigate the location of exosomes. $* * P<0.01$ compared with MKN45-exo. 
A
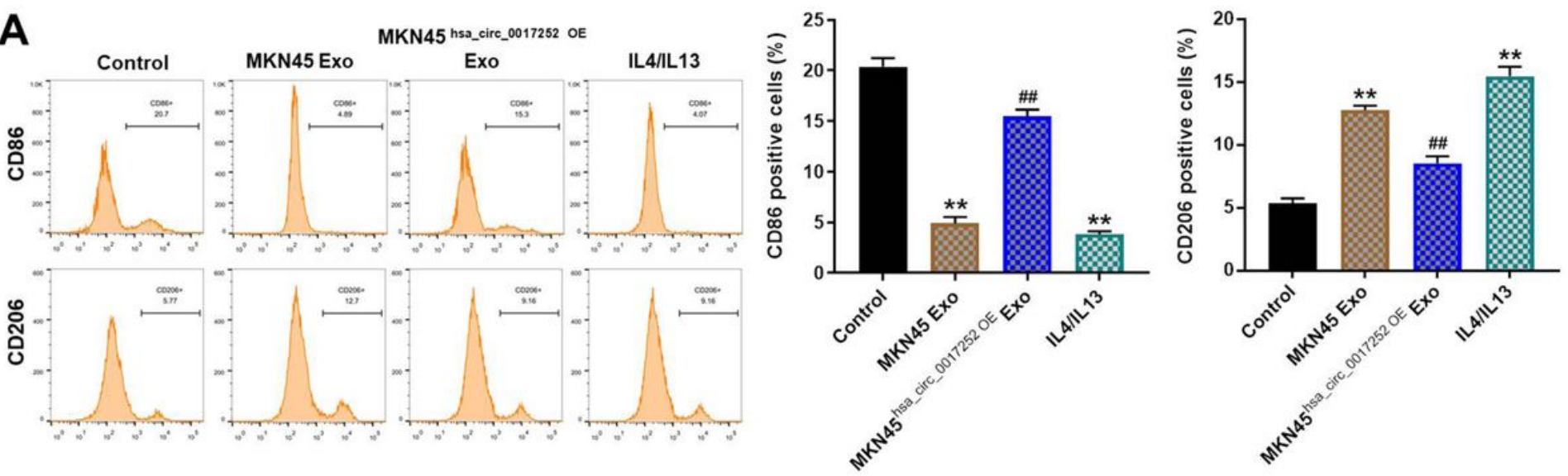

B

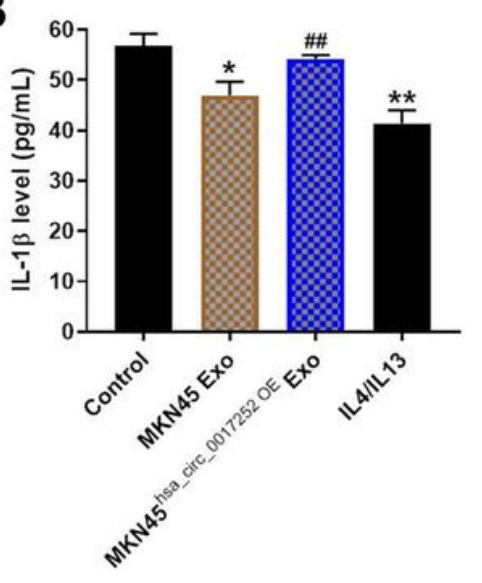

C

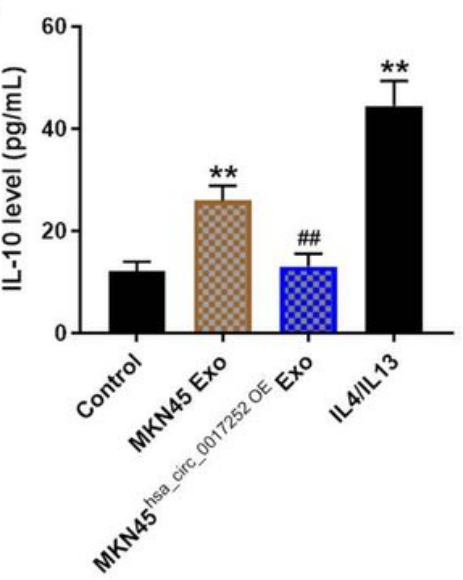

Figure 5

Exosomal hsa_circ_0017252 inhibited M2 polarization in macrophages. (A) Macrophages were added with MKN45-exo, MKN45 hsa_circ_0017252-OE-exo or IL-4/IL-13 (20 ng/ml) for $24 \mathrm{~h}$. Flow cytometry was used to detect CD206 or CD86 distribution rate in macrophages. (B, C) IL-1 $\beta$ and IL-10 levels in macrophage supernatants were assessed by ELISA. $* \mathrm{P}<0.05$, $* * \mathrm{P}<0.01$ compared with control. \#\#P<0.01 compared with MKN45-exo. 
A

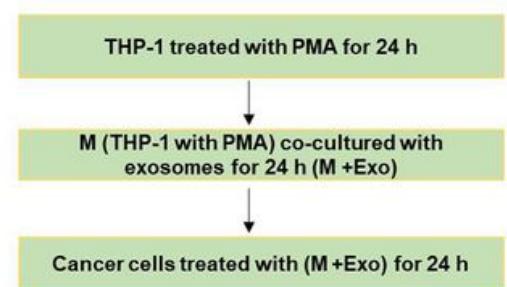

C
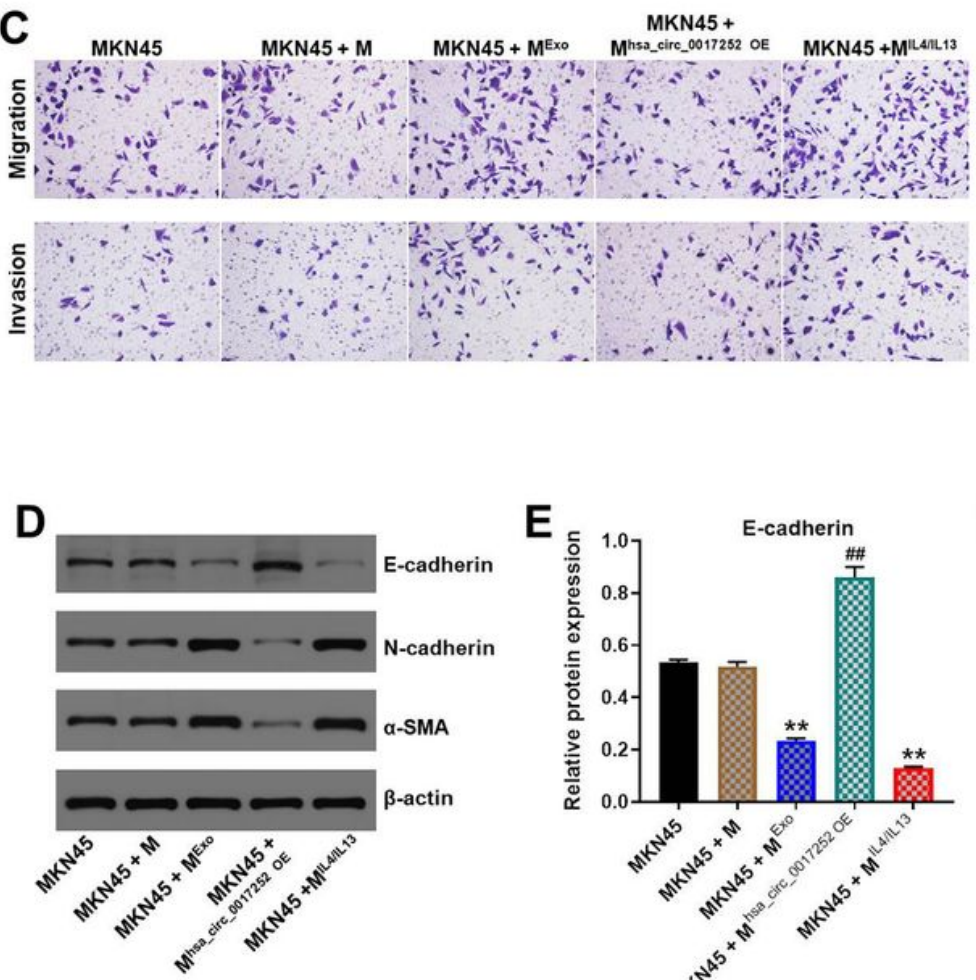

B

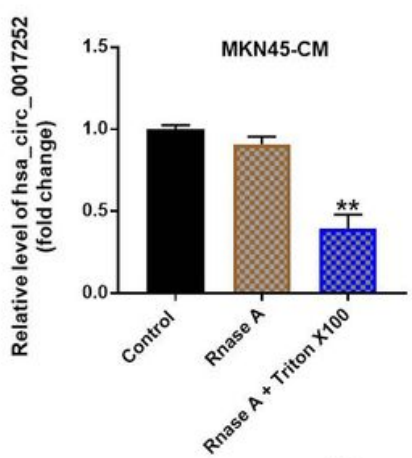

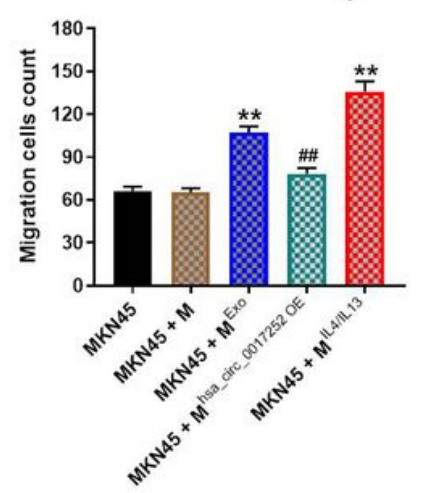

$\mathbf{F}$

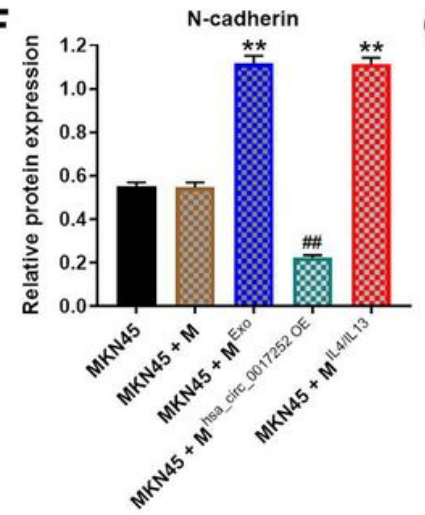

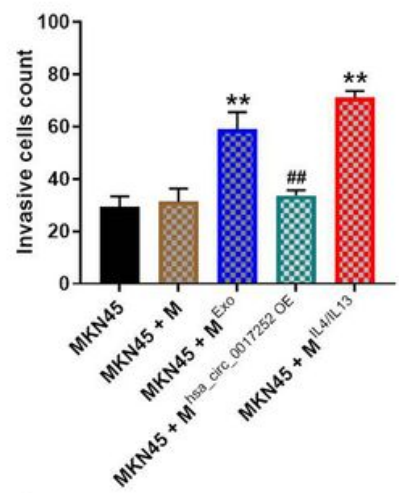

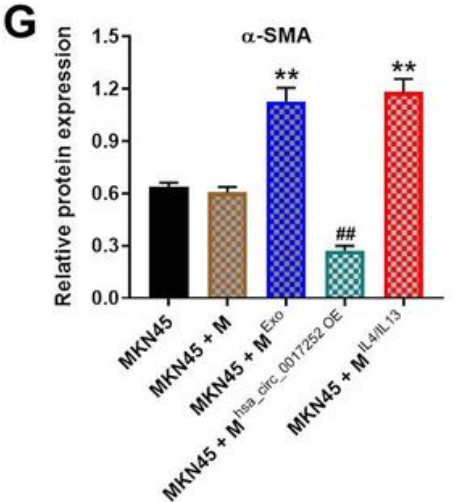

Figure 6

Exosomal hsa_circ_0017252 suppressed GC cell invasion and migration via inhibiting M2 macrophage polarization. (A) THP-1 cells were treated with PMA $(100 \mathrm{ng} / \mathrm{ml})$ for $24 \mathrm{~h}$, and then treated with exosomes derived from MKN45 cells. (B) The level of hsa_circ_0017252 in CM of macrophages/hsa_circ_0017252 was examined by RT-qPCR. (C) MKN45 cells were co-cultured with macrophages, Mexo, Mhsa_circ_0017252-exo or MIL-4/IL-13. GC cell invasion and migration was investigated by transwell assay. (D-G) N-cadherin, E-cadherin and a-SMA levels in GC cells were investigated by western blot. $\beta$ actin was used for normalization. **P<0.01 compared with control or MKN45. \#\#P<0.01 compared with Mexo. 
A

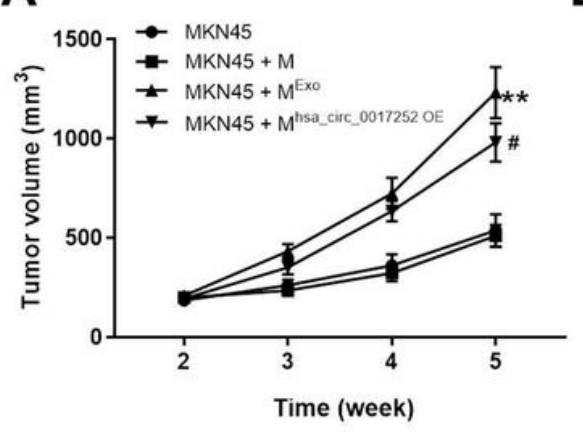

B

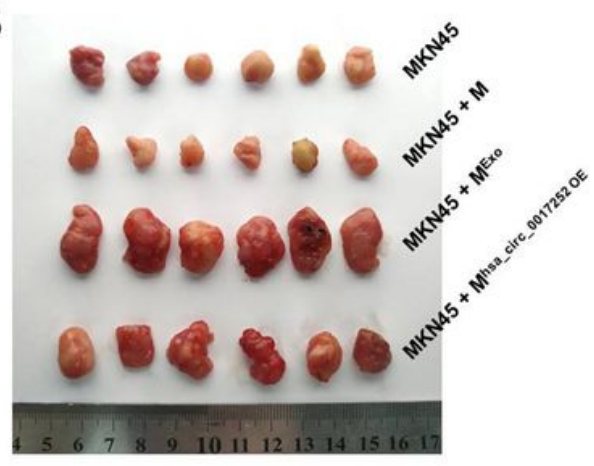

C

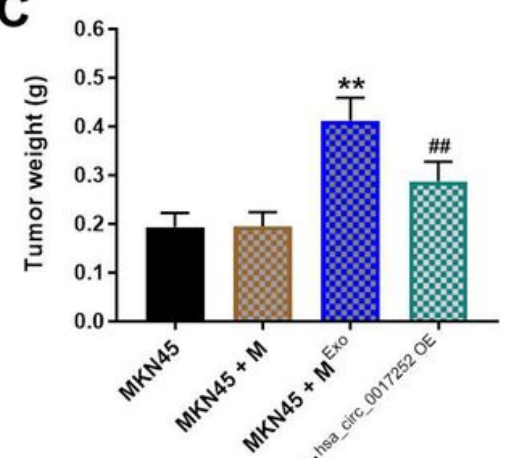

D

MKN45

MKN45 + M

MKN45 + MExo

MKN45 +
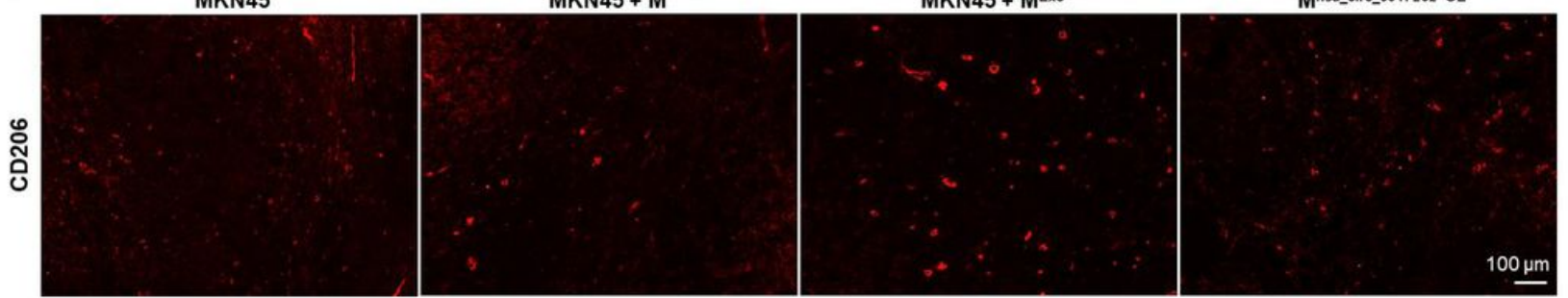

E
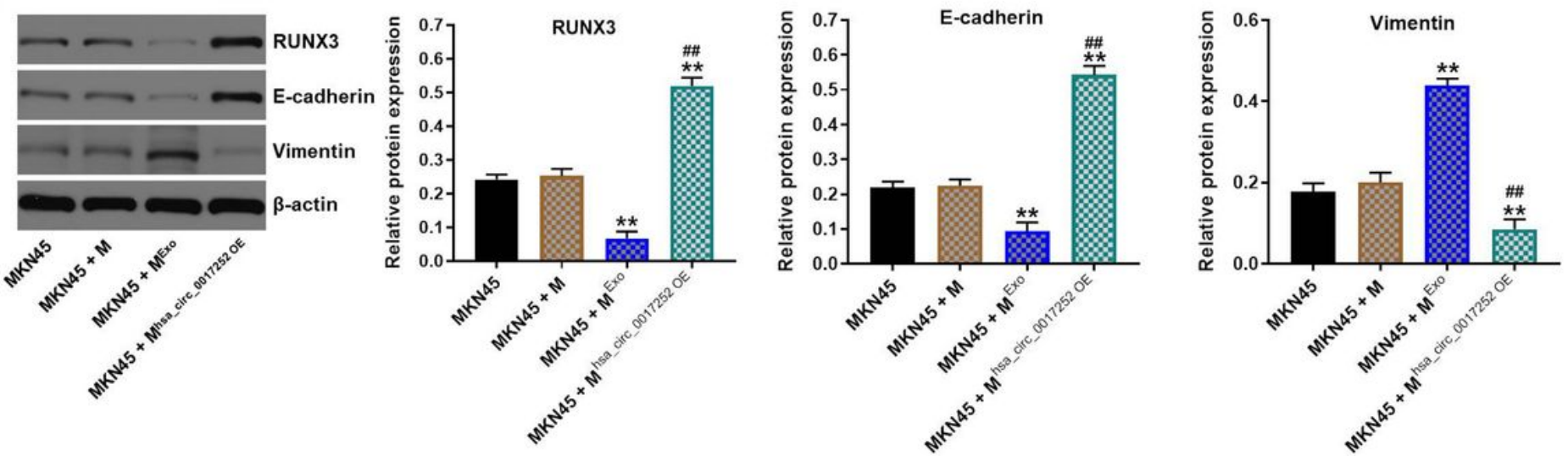

Figure 7

Exosomal hsa_circ_0017252 attenuated the growth of GC in vivo by decreasing the M2 polarization in macrophage. (A) The tumor volume of mice was calculated. (B) The tissues of mice were collected. (C) The weights of tumors in mice were recorded. (D) CD206 level in mice was detected by immunofluorescence staining. (E) E-cadherin, RUNX3 and vimentin levels in mice were tested by western blot. $\beta$-actin was used for normalization. **P<0.01 compared to MKN45, \#\#P<0.01 compared to MKN45 + Mexo.

\section{Supplementary Files}

This is a list of supplementary files associated with this preprint. Click to download.

- SupplementaryFigure1.jpg 